\title{
Cytogenetic Analysis of 13 Iranian Women with Premature Ovarian Failure
}

\section{A R T I C L E I N F O}

\section{Article Type}

Case Report

\section{Authors}

Hadipour F. ${ }^{1} M D$,

Hadipour Z. ${ }^{1} M D$,

Mousavi F. ${ }^{2} M S c$,

Bagherizadeh I. ${ }^{2} M S c$,

Shafaghatiti $\underline{Y}^{3} M \underline{M}$,

Behjati F* $P h D$

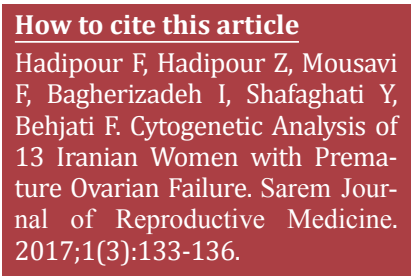

*"Sarem Cell Research Center (SCRC), Sarem Cell Research Center (SCRC), Medical Genetics Department, Sarem Women's Hospital" and "Genetic Reaearch Center, University of Welfore \& Rehabilitation Scienws", Tehran, Iran

${ }^{1}$ Sarem Fertility \& Infertility Research Center (SAFIR) and Sarem Cell Research Center (SCRC) and Medical Genetics Department, Sarem Women's Hospital, Tehran, Iran ${ }^{2}$ Sarem Cell Research Center (SCRC) and Medical Genetics Department, Sarem Women's Hospital, Tehran, Iran

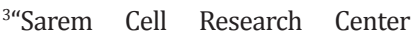
(SCRC), Sarem Cell Research Center (SCRC), Medical Genetics Department, Sarem Women's Hospital" and "Genetic Reaearch Center, University of Welfore \& Rehabilitation Scienws", Tehran, Iran

\section{Correspondence}

Address: Sarem Women's Hospital, Basij Square, Phase 3, Ekbatan Town, Tehran, Iran. Postal Code: 1396956111

Phone: +98 (21) 44670888

Fax: +98 (21) 44670432

f_behjati@uswrac.ir

\section{Article History}

Received: March 2, 2016

Accepted: June 21, 2016

ePublished: August 15, 2017

\section{A B S T R A C T}

Patients Information Considering the clinical importance and the deep impact of Premature Ovarian Failure (POF) on the life of affected people and the important role of genetics in its development, this study was conducted to determine the frequency and type of chromosomal abnormalities in Iranian women referred to Sarem Hospital with premature ovarian failure. Karyotype analysis and its association with phenotype were performed on 13 Iranian women with confirmed premature ovarian failure. The metaphase chromosomes were prepared and analyzed with G-bonding technique and mosaicism of 100 cells from lymphocyte cells was studied. Finally, chromosomal abnormalities were diagnosed in 2 of 13 patients (15.38\%) with premature ovarian failure. A patient with chromosomal mosaicism was [42] XX, 46 / [8] X, 45. The second patient had a translocation between chromosome $X$ and chromosome 9 , that was 46, XX, t(X,9)(q22.1;q22,1).

Conclusion The overall prevalence of chromosomal abnormalities is $15.38 \%$ among patients with premature ovarian failure, which is confirmed by our findings in the chromosomal examination of these women.

Keywords Cytogenetics; Diffusion; Premature Ovarian Failure; Prevalence; Chromosomal Abnormality

\section{I T A T I O N L I N KS}

[1] Premature gonadal failure [2] Premature ovarian failure [3] Familial premature ovarian failure [4] Evidence for a genetic factor in the etiology of premature ovarian failure [5] Molecular cytogenetic studies of Xq critical regions in premature ovarian failure patients [6] Premature ovarian failure (POF) syndrome: Towards the molecular clinical analysis of its genetic complexity [7] An X;9 translocation, primary amenorrhea, and hypothalamic dysfunction [8] Balanced reciprocal $(X ; 9)$ translocation in a girl with primary amenorrhea [9] New syndrome of amenorrhea in association with hypergonadotropism and apparently normal ovarian follicular apparatus [10] The gonadotropin resistant ovary syndrome [11] Premature menopause due to a small deletion in the long arm of the $\mathrm{X}$ chromosome: A report of three cases and a review [12] Hypergonadotropichypogonadism in female patients with galactosemia [13] Three siblings with premature gonadal failure [14] Investigating the role of X chromosome breakpoints in premature ovarian failure [15] Cytogenetic analysis of 531 Chinese women with premature ovarian failure [16] Genetic abnormalities in Turkish women with premature ovarian failure [17] A study of hypergonadotropic secondary amenorrhea with cytogenetics [18] The cytogenetics of premature ovarian failure [19] Chromosome mosaicism in patients with recurrent abortion or premature ovarian failure 
يائسكى مرتبط است. اين علايم شامل گَرَرفتكى، خشكى وازينال،

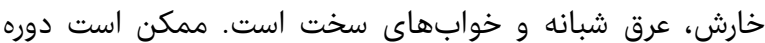

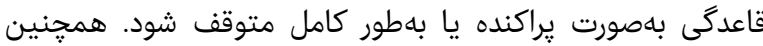

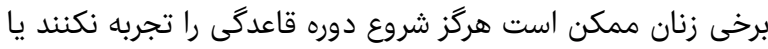

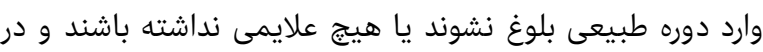

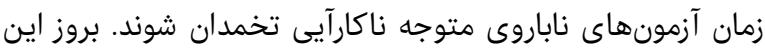

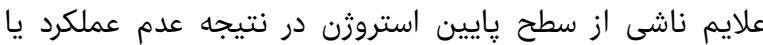

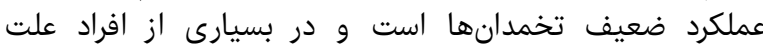

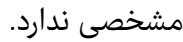
نارسايى زودرس تخمدان در بردي بردي از خانوادهها بسيار شايع است.

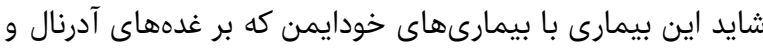

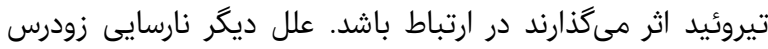

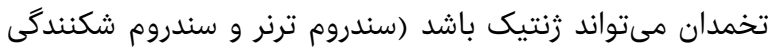

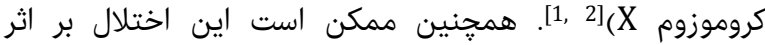

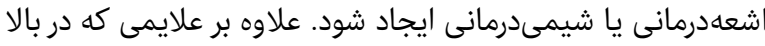

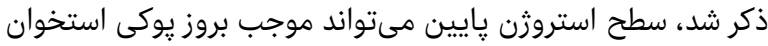

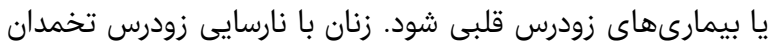

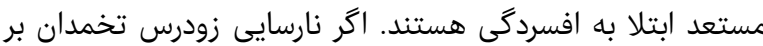

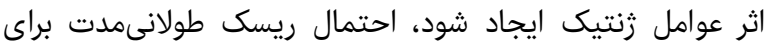

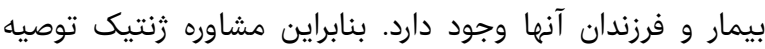

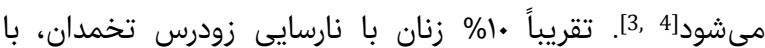

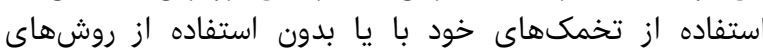

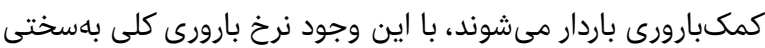

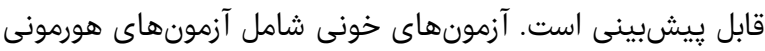

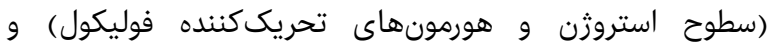

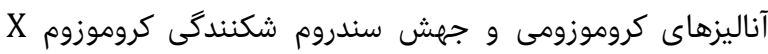

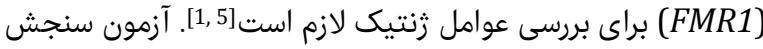
تراكم استخوان هم مىتواند مناسب باشد.

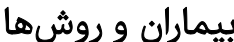

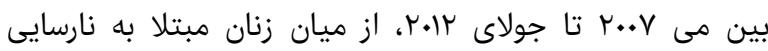

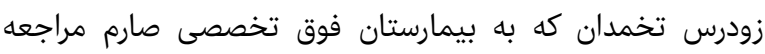

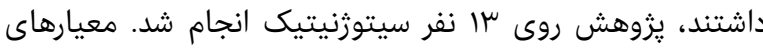

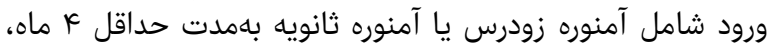

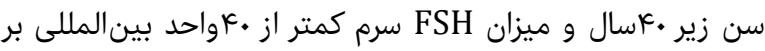

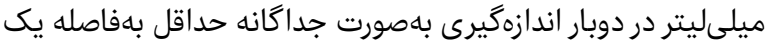

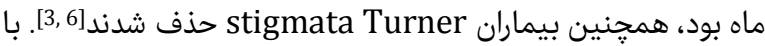

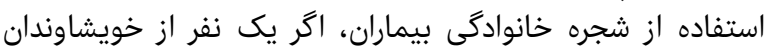

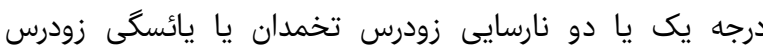

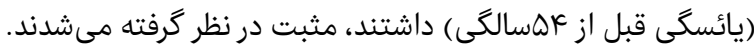

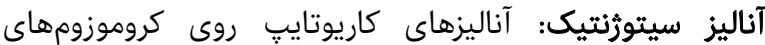

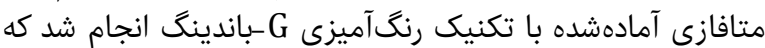

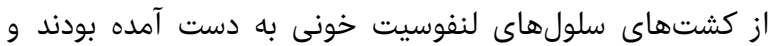

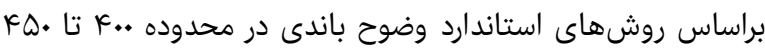

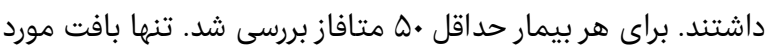

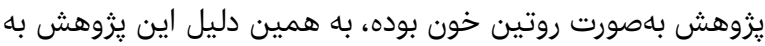

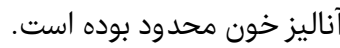

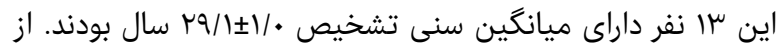

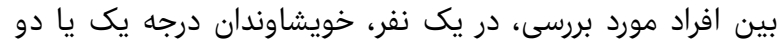

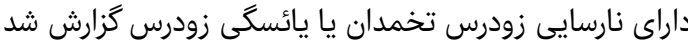

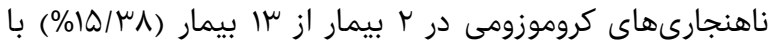

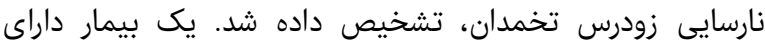

\section{آناليز سيتوزنتيك در سارسار سار زن بيمار ايرانى با نارسايى زودرس تخمدان}

MD فاطمه هادى تصيور

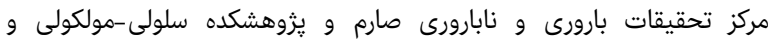

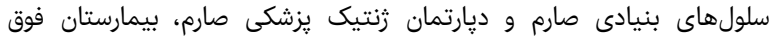
تخصصى صارم، تهران، ايران

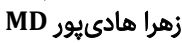

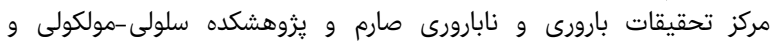

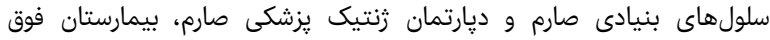
تخصصى صارم، تهران، ايران

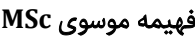

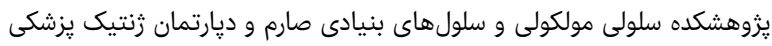
صارم، بيمارستان فوق تخصصى صارم، تهران، ايران مان

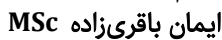

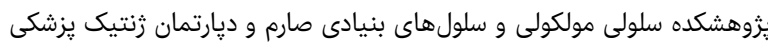

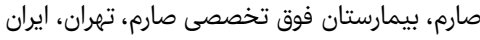
MD يوسف شفقتى بيمارستان فرون

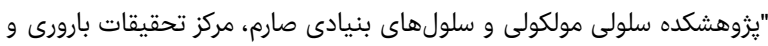

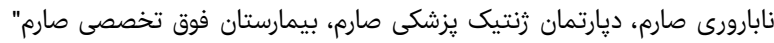

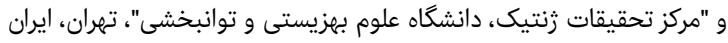

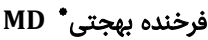

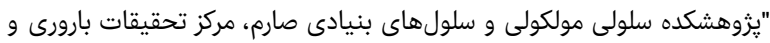

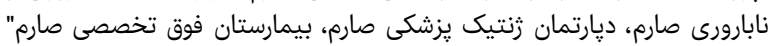

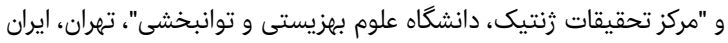

جكيده

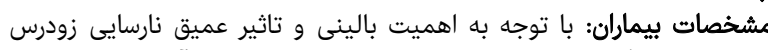

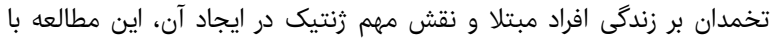

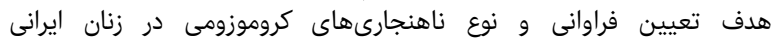

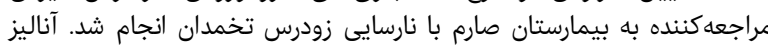

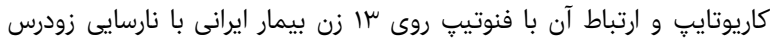

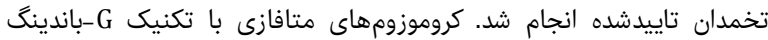

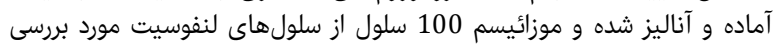

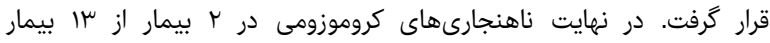

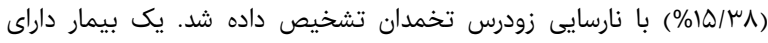

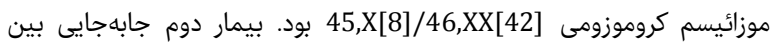

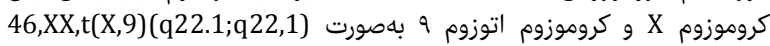

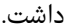

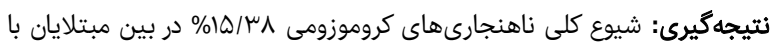

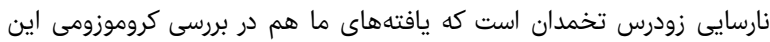

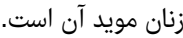

كليدوازهها: سيتوزنتيك، نارسايى زودرس تخمدان، شيوع، ناهنجارى كروموزومى

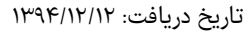

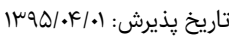

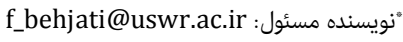

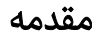

اكر تخمدانهاى يك زن قبل از •عاسالكى از كار بيفتد، فرد به زبه

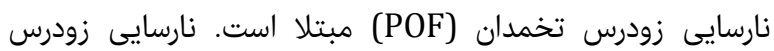

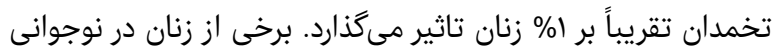

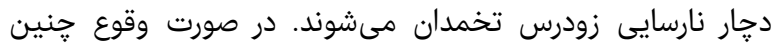

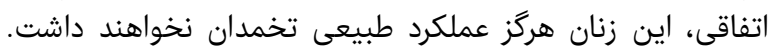

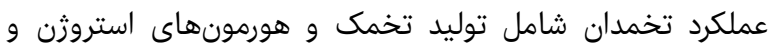

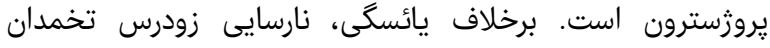

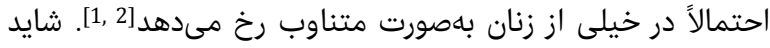

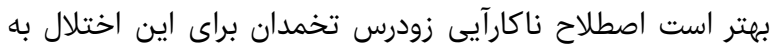

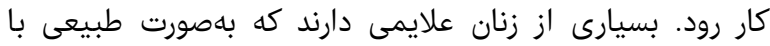


نتيجه

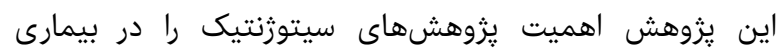

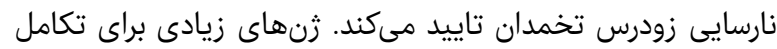

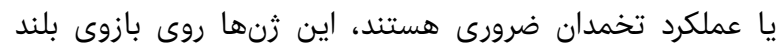

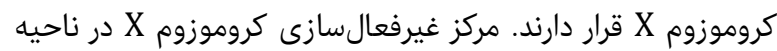

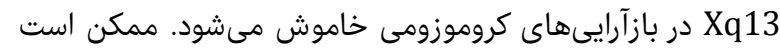

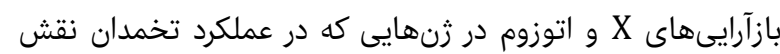

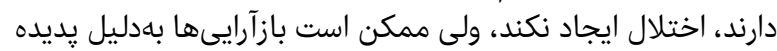

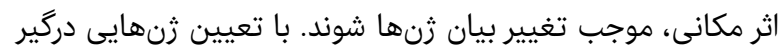

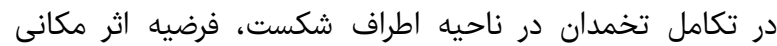

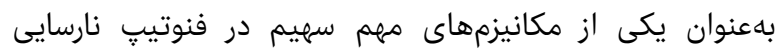
زودرس تخمدان معرفى مىشود.

تشكر و قدردانى: موردى از سوى نويسندكان ذكر نشده است.

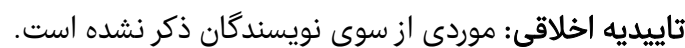
تعارض منافع: موردى وجود نداشته است إست

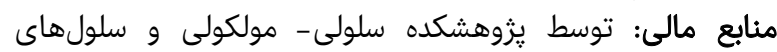
بنيادى صارم و مركز تحقيقات بارورى و نابارورى مارم تامين شده

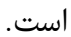

سهم نويسندكان: فاطمه هادئيور (نويسنده اول)، نكارنده

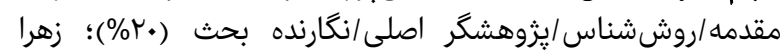

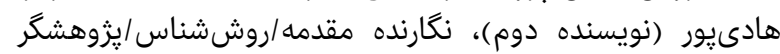

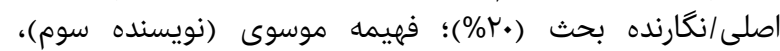

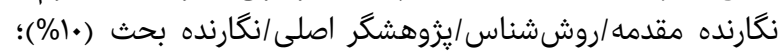

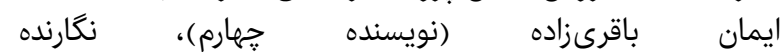

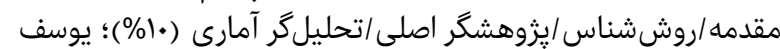

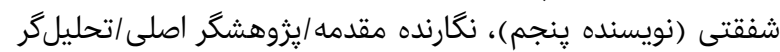

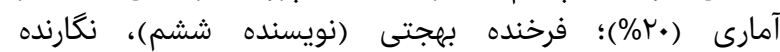

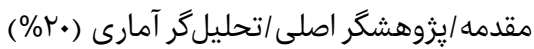

\section{منابع}

1- Coulam, CB. Premature gonadal failure. Fertil Steril. 1982;38(6):645-55.

2- de Moraes-Ruehsen M, Jones GS. Premature ovarian failure. Fertil Steril. 1967;18(4):440-61.

3- Mattison DR, Evans MI, Schwimmer WB, White BJ, Jensen B, Schulman JD. Familial premature ovarian failure. Am J Hum Genet. 1984;36(6):1341-8.

4- Coulam CB, Stringfellow S, Hoefnagel D. Evidence for a genetic factor in the etiology of premature ovarian failure. Fertil Steril. 1983;40(5):693-5.

5- Portnoi MF, Aboura A, Tachdjian G, Bouchard P, Dewailly D, Bourcigaux N, et al. Molecular cytogenetic studies of Xq critical regions in premature ovarian failure patients. Hum Reprod. 2006;21:2329-34.

6- Fassnacht W, Mempel A, Strowitzki T, Vogt PH. Premature ovarian failure (POF) syndrome: Towards the molecular clinical analysis of its genetic complexity. Curr Med Chem. 2006;13(12):1397-410.

7- Gardner HA, McConnon JK, MacKenzie MA. An X;9 translocation, primary amenorrhea, and hypothalamic dysfunction. Am J Med Genet. 1983;14(4):647-56.

8- Leddet-Chevallier I, Reid RA, Carrel RE, Sparkes RS. Balanced reciprocal $(X ; 9)$ translocation in a girl with primary amenorrhea. Ann genet. 1981;24(3):162-4.

9- Jones GS, DeMoraes-Ruehsen MA. New syndrome of amenorrhea in association with hypergonadotropism
موزائيسم كروموزومى [45,X[8]/46,XX[42 بودوم بوروم بيمار دوم

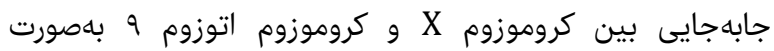
46,XX,t(X,9)(q22.1;q22,1)

جدول ) بروز جابجايى (X;9) با نارسايى زودرس تخمدان در جمعيتهاى مختلف جدول

\begin{tabular}{|c|c|}
\hline نواحى شكست & فنوتيبٍ \\
\hline $\mathrm{t}(\mathrm{x} ; 9)(\mathrm{q} 22 ; \mathrm{q} 12)$ & تاخير در بلوغ \\
\hline $\mathrm{t}(\mathrm{x} ; 9)(\mathrm{q} 22 ; \mathrm{q} 12)$ & دون وجود ديكر علايم بالينى سندروم ترنر \\
\hline $\mathrm{t}(\mathrm{x} ; 9)(\mathrm{q} 22.1 ; \mathrm{q} 22.1)$ & آمنوره اوليه بدون ساير علايم بالينى \\
\hline
\end{tabular}

بحث

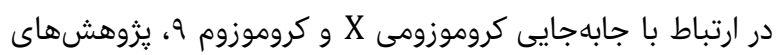

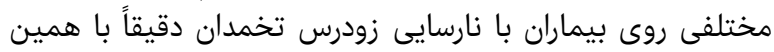

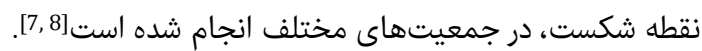

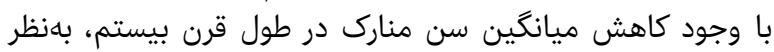

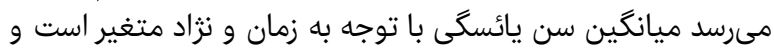

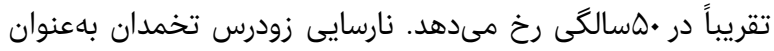

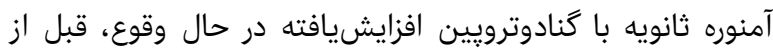

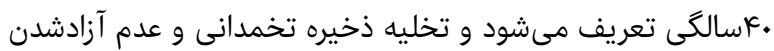

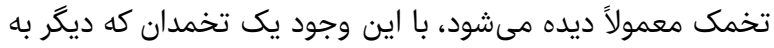

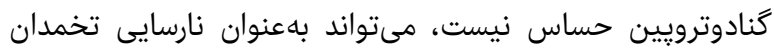

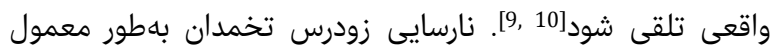

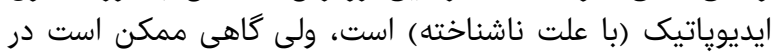

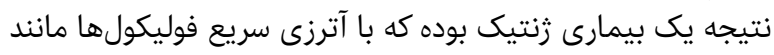

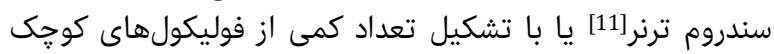

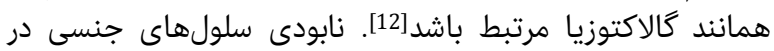

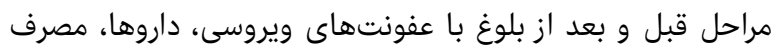

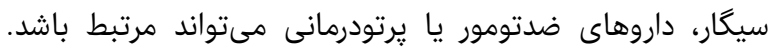

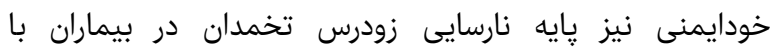

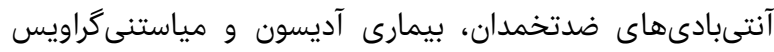

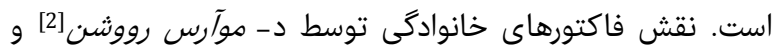

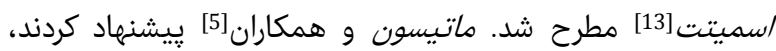

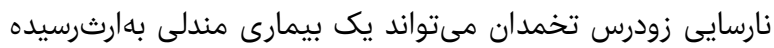

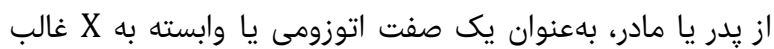

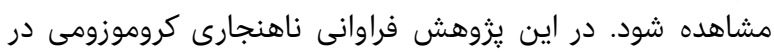

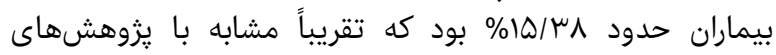

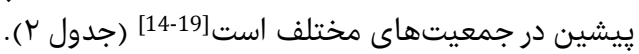

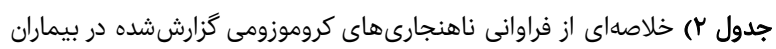

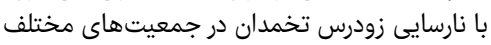

\begin{tabular}{|c|c|c|c|c|}
\hline فراوانى CAA & تعداد CA & حجم نمونه & مشخصه بالينى & جمعيت \\
\hline $1 / 4 \wedge$ & k & req & POF & يتاليايى \\
\hline $\mid \pi / 1$ & 4) & $\Delta \mu_{1}$ & POF & هينى \\
\hline YK & 11 & $V \Delta$ & POF & زرى (آناتولى) \\
\hline $1 \% / 0$ & سו & $1 . k$ & POF & جينى لجي \\
\hline$\mu r$ & ir & KV & POF & شيليايى \\
\hline $\mid Q / \mu \Lambda$ & $r$ & سו & POF & 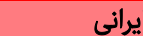 \\
\hline
\end{tabular}

*CA: chromosomal abnormalities

تعيين جزييات سيتوزنتيك بيماران جديد با فنوتيڤ نارسايى

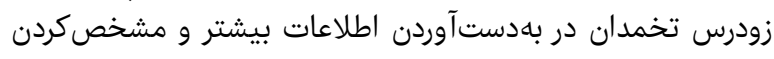

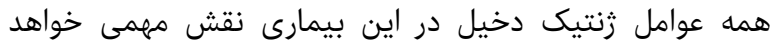
داشت. 
2012;5:32

15- Jiao X, Qin C, Li J, Qin Y, Gao X, Zhang B, et al. Cytogenetic analysis of 531 Chinese women with premature ovarian failure. Hum Reprod. 2012;27(7):2201-7.

16- Ceylaner G, Altinkaya SO, Mollamahmutoglu L, Ceylaner S. Genetic abnormalities in Turkish women with premature ovarian failure. Int J gynaecol Obstet. 2010;110(2):122-4.

17- Zhang J, Tang YY, Guo YP. A study of hypergonadotropic secondary amenorrhea with cytogenetics. J Chongqing Med Univ. 2003;2:151-152.

18- Castillo S, Lopez F, Tobella L, Salazar S, Daher V. The cytogenetics of premature ovarian failure. Rev Chil Obstet Ginecol. 1992;57(5):341-5. [Spanish]

19- $\mathrm{Wu} \mathrm{RC}$, Kuo PL, Lin SJ, Liu CH, Tzeng CC. chromosome mosaicism in patients with recurrent abortion or premature ovarian failure. J Formos Med Assoc. 1993;92(11):953-6.
عسار فاطمه هادىيور و همكاران and apparently normal ovarian follicular apparatus. Am J Obstet Gynecol. 1969;104(4):597-600.

10- Maxson WS, Wentz AC. The gonadotropin resistant ovary syndrome. Semin Reprod Endocr. 1983;1(2):14760.

11- Fitch N, De Saint Victor J, Richer CL, Pinsky L, Sitahal S. Premature menopause due to a small deletion in the long arm of the $\mathrm{X}$ chromosome: A report of three cases and a review. Am J Obstet Gynecol. 1982;142(8):968-72. 12- Kaufman FR, Kogut MD, Donnell GN, Goebelsmann U, March C, Koch R. Hypergonadotropichypogonadism in female patients with galactosemia. $\mathrm{N}$ Engl J Med. 1981;304(17):994-8.

13- Smith A, Fraser IS, Noel M. Three siblings with premature gonadal failure. Fertil Steril. 1979;32(5):52830.

14- Baronchelli S, Villa N, Redaelli S, Lissoni S, Saccheri F, Panzeri $\mathrm{E}$, et al. Investigating the role of $\mathrm{X}$ chromosome breakpoints in premature ovarian failure. Mol Cytogenet. 